\title{
Stabilization and Commitment: Forward Guidance in Economies with Rational Expectations
}

\author{
Andrew Hughes Hallett \\ George Mason University, and University of St Andrews \\ Phone: 1-703-993-2280 or 44-1334-462420 \\ Email: ahughesh@gmu.edu \\ Nicola Acocella \\ "Sapienza" University of Rome \\ Phone: +3906 49766359 \\ Fax: +39064957606 \\ Email: nicola.acocella@uniroma1.it
}




\section{Forward Guidance in Economies with REs}

Address correspondence to: Nicola Acocella, "Sapienza" University of Rome, Via Castro

Laurenziano 9, 00161 Rome, Italy, email: nicola.acocella@uniroma1.it 


\begin{abstract}
We construct a theory of forward guidance in economic policy making in order to provide a framework to explain the role and strategic advantages of including forward guidance as an explicit part of policy design. We do this by setting up a general policy problem in which forward guidance plays a role, and then examine the consequences for performance when that guidance is withdrawn. Following Acocella et al. (2013), who revisit the classical theory of economic policy in a world with rational expectations, we show that forward guidance provides enhanced controllability and stabilizability - especially where such properties may not otherwise be available. As a bi-product we find that forward guidance limits the scope and incentives for time inconsistent behavior in an economy whose policy goals are ultimately reachable. It also adds to the credibility of a set of policies therefore.
\end{abstract}

JEL Classifications: E42, E58, E61, E63

Keywords: Managing Expectations, Stabilizability, Dynamic Controllability, Time Consistency. 


\section{Introduction}

John Williams, President of the San Francisco Federal Reserve and member of the Fed's Open Market Committee, has argued that forward guidance and large scale asset purchases (popularly known as Quantitative Easing) are now the leading and most important forms of unconventional monetary policy (Williams, 2011). Both techniques were used extensively to engineer a recovery from the Great Financial Crisis of 2008-12, in the United States and elsewhere. ${ }^{1}$

But, whereas Quantitative Easing has been studied in some detail ${ }^{2}$ and is comparatively well understood in terms of how it is supposed to work, if not in terms of how large an impact it has had in practice, forward guidance has been equally widely used but with little understanding of how and in what circumstances it can work successfully, what the drawbacks might be, and what its impact might be. In short, we lack a proper analysis of the strategic value of forward guidance as a tool of monetary policy - both in general and in difficult circumstances.

Forward guidance may be defined as announcements made about the future stance of policy with the intention of influencing or managing expectations of future policy interventions, and hence the expected path of future outcomes for the economy. ${ }^{3}$ It may be unconditional and/or indefinite ("interest rates will remain low for a considerable period of time"); or conditional ("interest rates will remain low until unemployment falls below 6.5\%") or time dependent ("for two years"). ${ }^{4}$ It can be applied to achieve either controllability (the intention to achieve certain target values at a specified date in the future), or stabilizability (the ability to stabilize the economy about a certain path, be it one which leads to the desired target values or a continuation of the current policies, whatever shocks may yet emerge).

\footnotetext{
${ }^{1}$ Prominent examples, in the recent past, have been the UK, Canada, Australia, New Zealand, and Sweden.

${ }^{2}$ Williams (2011), Ugai (2007), Gagnon et al.(2011), Joyce et al. (2012), and referencese therein.

${ }^{3} \mathrm{We}$ do not consider forward guidance in the form of announcements made about the policy maker's projections on the future path of the economy here, although that is always possible and is indeed implied by the projections of the policy instruments - given access to a model of the economy and a matching information set.

${ }^{4}$ See Acocella and Hughes Hallett (2014).
} 
Forward guidance is therefore designed to make the private sector's expectations consistent with the policy intentions of the central bank or government with respect to interest rates, growth and investment - especially at the zero lower bound (Coeuré, 2013). There is some evidence that it has reduced the volatility of expectations (Coeuré 2013; Filardo and Hofmann 2014). On that view, the Governor of the Bank of Canada has argued that forward guidance is best used for stabilization, especially at the zero lower bound. ${ }^{5}$

The literature on forward guidance is, at this point, rather limited and restricted to a few specific problems or circumstances. A natural concern is whether forward guidance statements have had a perceptible impact on expectations. Kool and Thornton (2012) argue that they have not, at least not in the context of monetary policy in four leading OECD economies. On the other side, Campbell et al. (2012) argue that they did in the US when viewed over a longer sample period (1990-2011). Then again, Del Negro et al. (2013) suggest that our standard models often overestimate the size of these impacts. Obviously the jury is still out on that question.

Unsurprisingly given recent history, much of the theoretical work has been done in the context of interest rates being stuck at their zero lower bound. Here, for example, Gavin et al. (2013) find forward guidance announcements have the power to lower interest rates, prompting consumption and output to recover, if private sector expectations adjust - more so, the longer the horizon. However, in an important qualification, Levin et al. (2010) warn that the stability of the economy may be at risk. They find that, given moderate negative shocks, forward guidance can be used to stabilize the system, but conjecture that large negative shocks (such as would appear in a big recession) will overwhelm the forward guidance effects and leave us unable to stabilize the economy. But whether that is a result of large shocks, or of insufficiently responsive policies or inadequately chosen forward guidance, is a moot point. These results prompt another line of thought: that the effectiveness of forward guidance may depend on the form of guidance offered specifically whether it is Delphic (the expectations offered are in terms of outcomes; such as would be the case when trying to escape a serious recession), or Odyssean (the

\footnotetext{
${ }^{5}$ Stephen Poloz, interview in the Financial Times, 15 October 2014.
} 
expectations offered are in terms of a policy rule, or contingency plans defining how the authorities will react to certain conditions). ${ }^{6}$ This mirrors the unconditional vs. conditional forward guidance, with an exit strategy, distinction made in Acocella and Hughes Hallett (2014).

In trying to construct a theory of forward guidance, any formal analysis must fit within a general theory of policy announcements (Hughes Hallett and others 2012a,b; Acocella et al. $2014)^{7}$. Any such theory needs an understanding of the strategic advantages offered by forward guidance, its role in the policy arsenal, its value in terms of overall economic performance, and whether the expectations generated would be sustained or dissipated by time inconsistent revisions. That is the subject of this paper.

Starting from the framework in Acocella et al. (2013), we proceed by examining the advantages of policy rules which contain forward guidance, and then explore what is lost if those guidance terms are removed. Section 2 presents a generic economic model with rational expectations. Section 3 deals with conditions for controllability of this model, while Section 4 derives the corresponding conditions for stabilizability. Section 5 illustrates these concepts using two simple examples to explain the role of forward guidance; and underline the value of providing forward guidance in a policy rule where the private sector has, and reacts to, rational expectations of future outcomes. This leads to conclusions rather different from those appearing elsewhere in the existing literature (Section 6).

\section{Economic Models with Forward Looking Expectations}

Without loss of generality, we can write the generic linear RE model in its reduced form for a single policy authority, as follows:

$$
y_{t}=A y_{t-1}+B u_{t}+C y_{t+1 \mid t}+v_{t} \quad \text { for } t=1, \ldots, T .
$$

where $y_{t+1 \mid t}=E\left[y_{t+1} \mid \Omega_{t}\right]$ denotes the mathematical expectation of $y_{t+1}$ conditional on $\Omega_{t}$ (a common information set available to all agents at $t$ ) and $u_{t}$ is a vector of $m$ control

\footnotetext{
${ }^{6}$ For this distinction, see Raskin (2013) and Contessi and Li (2013), respectively.

${ }^{7}$ See Amato et al. (2002), or Brand et al. (2010) for further evidence.
} 
variables in the hands of the policymakers. The matrices $A, C$ and $B$ are constant and of order $S, S$, and $S \times m$, respectively, and have at least some elements which are nonzero. In this representation, $y_{0}$ is a known initial condition, and $y_{T+1 \mid 1}$ is some known, assumed or expected terminal condition (most probably one that describes the economic system's long run equilibrium state); ${ }^{8}$ and both are part of each information set $\Omega_{t}$. Note that the values of $u_{t}$ are not part of $\Omega_{t}$ since they are determined by policymakers.

Finally $v_{t}$ is a vector of exogenous shocks or other influences on $y_{t}$, with known mean but which comes from an unspecified probability distribution. Note also that the policy authority may have $q \leq \mathrm{S}$ explicit targets, but that the $m$ instruments are assumed to be linearly independent. Thus, $y \in R^{S}$ and $u \in R^{m}$.

This model can now be solved from the perspective of any particular period, say $t=1$, by putting it into its final form conditional on the information available in that period:

$$
\left(\begin{array}{c}
y_{1 \mid 1} \\
: \\
: \\
: \\
y_{T \mid 1}
\end{array}\right)=\left[\begin{array}{ccccc}
I & -C & 0 & \cdot & 0 \\
-A & I & & \cdot & \cdot \\
0 & & . & & 0 \\
\cdot & \cdot & & \cdot & -C \\
0 & \cdot & 0 & -A & I
\end{array}\right]^{-1}\left\{\left[\begin{array}{ccccc}
B & 0 & \cdot & \cdot & 0 \\
0 & \cdot & & & \cdot \\
\cdot & & \cdot & & \cdot \\
\cdot & & & \cdot & 0 \\
0 & . & . & 0 & B
\end{array}\right]\left(\begin{array}{c}
u_{|| 1} \\
\cdot \\
\cdot \\
\cdot \\
u_{T \mid 1}
\end{array}\right)+\left(\begin{array}{c}
v_{1 \mid 1} \\
\cdot \\
\cdot \\
\cdot \\
v_{T \mid 1}
\end{array}\right)+\left(\begin{array}{c}
A y_{0} \\
0 \\
\cdot \\
\cdot \\
0
\end{array}\right)+\left(\begin{array}{c}
0 \\
\cdot \\
\cdot \\
0 \\
C y_{T+|| 1}
\end{array}\right)\right\}
$$

Although equation (1) has been solved from the point of view of $\Omega_{1}$, it is understood that it could be derived for each $\Omega_{t}, t=1, \ldots, T$, in turn, where $y_{j \mid t}=E_{t}\left(y_{j}\right)$ if $j \geq t$, but $y_{j \mid t}=y_{j}$ if $j<t$; similarly for $u$ and $v$.

The equation to which (2) is the solution makes it clear that neither policymakers, nor the private sector are required to make expectation errors for the policies to work as planned. In fact, equation (3) below shows just the opposite: those expectations are exactly consistent with what the private sector expects the outcomes to be. It then only remains to discover if

\footnotetext{
${ }^{8}$ There is no indeterminacy problem here. The dynamic conditions which guarantee the existence of a solution are automatically satisfied, given any particular information set, if the inverse in (2) exists - which we show to be true below. Given that inverse, Hughes Hallett and Fisher (1988) show that the saddle point property (that the system has the correct number of stable and unstable roots to ensure a solution, Blanchard and Khan (1980)) is satisfied. One implication is that it no longer matters what the value of the terminal condition is (or if none is specified) so long as the policy horizon is far enough away $(T \rightarrow \infty)$. Indeterminacy may however follow for smaller values of $T$ if $y_{T+1 / 1}$ cannot be specified.
} 
it is possible to shift expectations in such a way that the economy's outcomes can reach certain specified target values at certain points of time.

It is easy to show that this final form solution always exists since the inverse matrix in (2) is well defined provided the matrix product $A C$ does not contain a unit root (Hughes Hallett et al, 2012b).

\section{Controllability}

We can now write the model in final form in the following way:

$$
\left(\begin{array}{c}
y_{1 \mid 1} \\
\cdot \\
\cdot \\
\cdot \\
y_{T \mid 1}
\end{array}\right)=\left[\begin{array}{ccccc}
R_{11} & \cdot & \cdot & \cdot & R_{1 T} \\
\cdot & \cdot & & \cdot \\
\cdot & & \cdot & & \cdot \\
\cdot & & & \cdot & \cdot \\
R_{T 1} & \cdot & \cdot & \cdot & R_{T T}
\end{array}\right]\left(\begin{array}{c}
u_{1 \mid 1} \\
\cdot \\
\cdot \\
\cdot \\
u_{T \mid 1}
\end{array}\right)+\left(\begin{array}{c}
b_{1 \mid 1} \\
\cdot \\
\cdot \\
\cdot \\
b_{T \mid 1}
\end{array}\right) \text {, or } y=R u+b
$$

where $R=T_{T}^{-1}(I \otimes B), b=T_{T}^{-1}\left\{E\left(v \mid \Omega_{1}\right)+\left(A^{\prime}: 0\right)^{\prime} y_{0}+\left(0: C^{\prime}\right) y_{T+1 \mid 1}\right\}, \quad$ and $\otimes \quad$ denotes a Kronecker product. In this representation, each $R_{t, j}=\partial y_{t \mid 1} / \partial u_{j \mid 1}$ is an $S \times m$ matrix of policy multipliers for $t, j=1, \ldots, T$. Notice that $R_{t, j} \neq 0$ even if $t<j$. Hence equation (3) implies that $R_{t, j}$ is a matrix of conventional policy multipliers between $y_{t \mid 1}$ and $u_{j \mid 1}$, with a delay of $t-j$ between implementation and realization if $t \geq j$. But if $t<j$, then $R_{t, j} \neq 0$ represents a matrix of anticipatory effects, on $y_{t \mid 1}$, of an announced or anticipated policy change $u_{j \mid 1}$ at some point in the future.

\section{Multi-period static controllability}

Static controllability defines the set of conditions which must hold if an arbitrary set of target values can be achieved for the endogenous variables $y_{t}$ in each period. Define those target values to be $\bar{y}_{t \mid 1}$; and $\bar{y}$ the corresponding stacked vector of those desired values across time periods. 
Static controllability, meaning the ability to reach desired values for the targets in each period, evidently requires the matrix $R$ in (3) to possess an inverse: ${ }^{9}$

(4) $u=R^{-1}(\bar{y}-b)$

where $y, u$ and $b$ are all understood to be expectations conditioned on the current information set $\Omega_{t}$, including the terminal condition, as specified in equation (3). Hence:

Theorem 1 (static controllability under REs). Under REs, static controllability by a single player, as in any conventional backwards looking model, requires as many independent policy instruments as there are target variables in each time period.

Proof. See Hughes Hallett et al. (2012b).

\section{Multi-period dynamic controllability}

A model is said to be dynamically controllable if a sequence of instrument values $u_{1}, \ldots, u_{t}$ can be found that will reach any arbitrary values, $\bar{y}_{t}$, for the target variables in period $t$ (in expectation) given an arbitrary starting point $y_{0}$. Starting from period 1 , dynamic controllability therefore requires a sequence of intended instrument values, $u_{1 \mid 1}, \ldots . ., u_{T \mid 1}$, that guarantee $\bar{y}_{t \mid 1}$ is reached in period $t$. Given an initial state $y_{0}$ and terminal condition $y_{T+1 \mid 1}$, this is possible only if the sequence of policy multipliers and anticipatory effects in the $t$-th row block of (3), [ $\left.R_{t, 1} \ldots . . R_{t, T}\right]$, is of full rank. That is, if $\mathrm{r}\left[R_{t, 1} \ldots R_{t, T}\right]=S$.

Theorem 2 (sufficient conditions for dynamic controllability with REs). The economy represent-ed here by (1) is dynamically controllable over the sub-interval $(1, t)$, when $T \geq S$ and when $t<T$, if $\mathrm{r}\left[R_{t, 1} \ldots . R_{t, S}\right]=S$.

Proof. See Hughes Hallett et al. (2012b).

\footnotetext{
${ }^{9}$ For convenience we have assumed that the number of instruments and targets does not vary over time.
} 
Comment 1. It is important to see why time inconsistency will not appear here. Controllability at period $t$ means that, barring unforeseen shocks, the policymaker will be able to reach his desired values for $y_{t}$ in expectation. Hence, $y_{t \mid t}=y_{t \mid 1}=\bar{y}_{t}$ are fixed or at least known quantities. But $y_{1 \mid t}=y_{1 \mid 1}$ is fixed by history; and $u_{1 \mid t}=u_{1 \mid 1}$ likewise. It is then easy to see that, if nothing else changes, $u_{t \mid t}=u_{t \mid 1}$. The policymaker is of course free to set $u_{t \mid t} \neq u_{t \mid 1}$. But he would never do so because $\bar{y}_{t}$ is his first best value and is reachable given no information changes or unforeseen shocks. Policy makers have no incentive, still less a strategic interest, in choosing to make themselves worse off than they need to be.

Could time inconsistency not emerge as new shocks appear over time? Comment 1 says that time inconsistency would never appear, and that will remain true unless: a) there are too few instruments to control the targets in any time period ( $m<S$ : static controllability doesn't apply at any $t$ ); and b) there are too few time periods between the current period, and the time at which the target values are to be achieved, for the available instruments to reach the number of targets remaining $(S / m<t$ where $t$ is the date by which the desired targets are to be attained: the rank condition for dynamic controllability no longer applies). Thus comment 1 remains true for all time periods. However, if $t$ remains fixed, the possibility of those conditions holding becomes larger as we go further into the policy period (i.e as $t-t_{c}$ gets smaller, where $t_{c}$ is the current period). But if that happens when the policy revisions are driven by shocks or new information, they are not an example of time inconsistency, but of contingency planning. No rational policymaker would fail to react to new information to correct his policy settings when there is new information or the old information was wrong. But we don't say they are being time inconsistent when they do that. It is just a normal feature of any feedback regime, including those without rational expectations and hence no chance of time inconsistent behavior.

\section{Stabilizability under Rational Expectations}

We can apply the reasoning underlying Theorem 2 to show that any economy can be stabilized to an arbitrary degree under rational, forward looking expectations if it is also dynamically controllable. An arbitrary degree of stabilization means that policy rules can 
be found to make the economy follow an arbitrarily stable path, based on an arbitrary set of eigenvalues, such that it returns to the original path following a shock (Wonham, 1974). ${ }^{10}$

Theorem 3 (stabilizability and REs). For any economy represented by (1), with arbitrary coefficient matrices $A, B$ and $C$, we can always find a series of dynamic forward-looking policy rules, $u_{t \mid 1}=\sum_{j=1}^{T} K_{t j} y_{j-1 \mid 1}+k_{t \mid 1},{ }^{11}$ such that the controlled economy is stabilizable up to an arbitrary set of eigenvalues, if that economy is dynamically controllable as defined in Theorem 2.

Proof $^{12}$ : Equation (1), with arbitrary coefficient matrices $A, B$ and $C$, can be reduced to its final form (2). Substituting the policy rule $u_{t \mid 1}=\sum_{j=1}^{T} K_{t j} y_{j-1 \mid 1}+k_{t \mid 1}$ for each $t=1, \ldots, T$ shows that the controlled economy will behave as:

$$
\left(\begin{array}{c}
y_{1 \mid 1} \\
\cdot \\
\cdot \\
\cdot \\
y_{T \mid 1}
\end{array}\right)=\left[\begin{array}{ccccc}
R_{11} & \cdot & \cdot & \cdot & R_{1 T} \\
\cdot & \cdot & & \cdot \\
\cdot & & \cdot & \cdot \\
\cdot & & & \cdot & \cdot \\
R_{T 1} & \cdot & \cdot & \cdot & R_{T T}
\end{array}\right]\left[\begin{array}{ccccc}
K_{1,1} & K_{1,2} & \cdot & \cdot & K_{1, T} \\
K_{2,1} & \cdot & & & \cdot \\
\cdot & & \cdot & & \cdot \\
\cdot & & & \cdot & K_{T-1, T} \\
K_{T, 1} & \cdot & \cdot & K_{T, T-1} & K_{T, T}
\end{array}\right]\left(\begin{array}{c}
y_{0} \\
y_{1 \mid 1} \\
\cdot \\
\cdot \\
y_{T-1 \mid 1}
\end{array}\right)+\left(\begin{array}{c}
c_{1 \mid 1} \\
\cdot \\
\cdot \\
\cdot \\
c_{T \mid 1}
\end{array}\right)
$$

where $y_{0 \mid 1}=y_{0}$ and $c_{t \mid 1}=b_{t \mid 1}+T_{T}^{-1} B k_{t \mid 1}\left(b_{t \mid 1}\right.$ was defined in (3)). Rewriting (5), we now have

$$
\tilde{y}_{t}=R K \tilde{y}_{t-1}+c
$$

where $\tilde{y}_{t}$ is the stacked vector on the left of (5).

For an economy to be stabilizable at $t$, it must possess the property that it would return to the initially expected path, whatever the initial conditions and shocks experienced up to that point, given no further shocks or changes in expectations appear (Wonham, 1974). This property will exist if the iteration matrix, $R K$, has its roots inside the unit circle. But we can go further. Any particular $y_{t \mid 1}$ will follow an arbitrarily stable path if we can pick $K_{t 1} \ldots . . K_{t T}$ to generate an arbitrary set of eigenvalues for that matrix for each $t$. Suppose we want to

\footnotetext{
${ }^{10}$ Theorem 3 gives the REs analogue of the standard theorem for backward looking, physical systems.

${ }^{11}$ Note that this rule, when used in period $t<T$, employs actions and anticipated actions up to period $T$.

${ }^{12}$ Adapted from Acocella et al. (2013, Chapter 12).
} 
choose iteration matrix $D=Z \Lambda Z^{-1}$, where $\Lambda$ is a diagonal matrix of chosen eigenvalues, and $Z$ is a matrix of corresponding eigenvectors. Then, as long as $T>S$ and the matrix $R$ has full rank $S T$ (i.e., $m \geq S$, so that static controllability applies), we can calculate the required $K$ from $K=R^{-1} Z \Lambda Z^{-1}$. But if $m<S$ and dynamic controllability applies (as in the theorem), then we can use a generalized left-inverse instead: $K=R^{+} Z \Lambda Z^{-1}$, with $R^{+}=\left(R^{\prime} R\right)^{-1} R^{\prime}$ as one obvious possibility. This generalized inverse always exists, given dynamic controllability, since $R^{\prime} R$ has full rank with $\mathrm{r}[R]=m T$ by Sylvester's inequality. To see this, recall $R=T_{T}^{-1}(I \otimes B)$ where $T_{T}^{-1}$ is a square $S T \times S T$ matrix of full rank and $(I \otimes B)$ is a block diagonal matrix with rank $m T$. Hence, by Sylvester's inequality, $\mathrm{r}[R] \geq S T+m T-S T$ $=m T$. But if $m<S$, then $\mathrm{r}[I \otimes B]$ cannot be greater than $m T$ by definition. Hence $\mathrm{r}[R]=m T$, which means that $\left(R^{\prime} R\right)^{-1}$ exists and that $R^{+}$is always available.

Comment 2. Note that the policy rules described in Theorem 3 are both forward and backward looking in that they react to expected future developments, including to the effects of these rules applied in the future, and to feedback from past outcomes (past "failures") - in exactly the same way as the private agents in the economy have been assumed to do.

Comment 3. Thus we can infer that a RE model which is dynamically controllable at $t=1$ in the sense of Theorem 2, is also stabilizable from $t=1$. Hence Theorem 3 generalizes on Wonham's theorem, where stabilizability can be achieved for the first time only in period $S$. However it is not generally possible to dispense with the feed-forward part of the policy rule for the obvious reason that it has to control both the feed-forward and feedback behavior of private agents.

Comment 4. The key lesson therefore is that, in models with forward looking behavior, the closed loop (as opposed to feedback) characteristics of our policy rules are of special importance. Closed loop means reacting to changes in expectations of future events as they appear, in addition to past outcomes as they deviate from plan. 
Comment 5. Theorem 3 delivers a different result from Levin et al. (2010), who conjecture (but do not prove) that forward guidance terms in the policy rule can be used to stabilize an economy in the face of small shocks, but not large shocks. But they do not allow policymakers freedom to choose the policy rules (and hence the policies) needed to stabilize the economy. Theorem 3 shows that such policy rules can always be so chosen if there are no further constraints. We examine this difference in greater detail elsewhere (Hughes Hallett and Acocella, 2014) and show that the capacity to stabilize the economy depends not on the size or direction of the shocks; but on the choice of parameters in the policy rule. Offsetting large shocks needs a stronger rule and hence more suitable forward guidance. Large negative shocks may prevent the Taylor principle being applied in some instances, but these are mostly cases close to the zero lower bound where that principle would be unsuitable anyway.

\section{An Illustration: Stabilizability, with and without forward looking policies.}

We now construct two simple examples of this stabilizability result to illustrate the importance of using forward looking policies and forward guidance given forward looking behavior by the private sector. These examples are constructed to explain a paper by Cochrane (2011) which claims that the Taylor rule in a New Keynesian model will produce results that are typically unstable. The claim is correct, but not for the reason offered. The correct reason is, no rational policymaker would ever attempt to use a backward looking policy rule to manage an economy with forward looking behavior or anticipations. Forward guidance is needed as well.

For the purposes of illustration, consider a one equation RE model with dynamics:

$$
y_{t}=a y_{t-1}+b y_{t+1}^{e}+c x_{t}+\varepsilon_{t}
$$

Such a model can be derived from a conventional New Keynesian model of the type used by Mishkin (2002), say, to assess the ability of Taylor rules to control inflation and the output gap. That is, we can start from:

$$
\pi_{t}=(1-\lambda) \beta E_{t} \pi_{t+1}+\lambda \pi_{t-1}+\kappa z_{t}+\phi f_{t}+v_{t}
$$




$$
z_{t}=\varsigma E_{t-1} z_{t}-\sigma\left(i_{t}-E_{t} \pi_{t+1}\right)+\chi f_{t}+\eta_{t}
$$

where (80) is an aggregate supply equation with dynamics, $\pi_{t}$ is the rate of inflation, $z_{t}$ the output gap; $f_{t}$ is the stance of fiscal policy, ${ }^{13} i_{t}$ the interest rate (monetary policy instrument), and $v_{t}$ and $\eta_{t}$ are random shocks. Eliminating $z_{t}$ between (8) and (9) yields:

$$
\pi_{t}=(1-\lambda) \beta E_{t} \pi_{t+1}+\lambda \pi_{t-1}-\kappa \sigma\left(i_{t}-E_{t} \pi_{t+1}\right)+\varepsilon_{t}
$$

where $\varepsilon_{t}=\kappa E_{t-1} z_{t}+(\kappa \chi+\phi) f_{t}+\kappa \eta_{t}+v_{t}$ represents a composite term of exogenous "shocks". This definition of $\varepsilon_{t}$ also involves an approximation in that $E_{t-1} z_{t}$ should, strictly speaking, be an endogenous (rational) expectation of the output gap. Classifying it as part of the composite error term is to recognize the reality that the private sector typically does not have full REs for the output gap; or cannot measure them accurately; or that the private sector is no more able to separate cyclical from structural changes in the trend of output than can the policymakers - the delay needed for the data to do so accurately being up to 4 years. ${ }^{14}$ Agents therefore typically use a simple forecasting or extrapolation device for $E_{t-1} z_{t}$ instead. Given that, we can recast (10) as a particular case of (7) with $a=\lambda$; $b=(1-\lambda) \beta+\kappa \sigma$; and $c=-\kappa \sigma$. In this case, $x_{t}$ is the policy instrument (interest rate); and $y_{\mathrm{t}}$ is the policy target (inflation).

We can now investigate the ability to stabilize, based on our notion of controllability, using Theorem 3. Consider now two different decision rules for managing (7):

a) with no forward looking elements,

$$
x_{t}=k_{1} y_{t-1}+k_{2} ; \text { and }
$$

b) with an added forward looking element,

$$
x_{t}=k_{1} y_{t-1}+d y_{t+1}^{e}+k_{2} \text { say. }
$$

\footnotetext{
${ }^{13}$ Many New Keynesian models specify marginal costs, $m c_{t}$, as the push factor in inflation, in place of $f_{t}$. In this case (10) would have $\kappa \chi f_{t}+\phi m c_{t}$ in $\varepsilon_{t}$ in place of the $(\kappa \chi+\phi) f_{t}$ term. This alternative specification would lead to a model of identical form to that specified here.

${ }^{14}$ See Hughes Hallett, Kattai and Lewis (2012).
} 
We substitute (11) or (12) into (7) to see the behavior of the economy under control in each case:

$$
\begin{aligned}
y_{t} & =\left(a+c k_{1}\right)+b y_{t+1}^{e}+\varepsilon_{t}^{*} ; \text { and } \\
y_{t} & =\left(a+c k_{1}\right) y_{t-1}+(b+c d) y_{t+1}^{e}+\varepsilon_{t}^{*}
\end{aligned}
$$

respectively, where $\varepsilon_{t}^{*}=\varepsilon_{t}+c k_{2}$. Renormalizing (13) and (14) on their lead terms, then taking expectations conditional on information available in period $t$, and dropping the superscript " $e$ " for simplicity, leaves us with two alternative models to be stabilized:

$$
\begin{aligned}
& y_{t+1}=b^{-1} y_{t}+b^{-1}\left(a+c k_{1}\right) y_{t-1}+b^{-1} \varepsilon_{t}^{*} \\
& y_{t+1}=(b+c d)^{-1} y_{t}+(b+c d)^{-1}\left(a+c k_{1}\right) y_{t-1}+(b+c d)^{-1} \varepsilon_{t}^{*}
\end{aligned}
$$

It is already obvious that (15) presents us with only one opportunity, via the choice of $k_{1}$, to choose the coefficients and thus the roots of the economy under control. Yet there are two roots. In fact, it will not be possible to stabilize this economy with a simple feedback rule at all, unless $b>1$, let alone to do so up to an arbitrary pair of eigenvalues.

Equation (16), by contrast, gives us the opportunity to choose two coefficients, and hence both characteristic roots in the economy under control, given our freedom to choose both $k_{1}$ and $d$. Hence, this system is stabilizable, and stabilizable up to an arbitrary set of eigenvalues.

We demonstrate these two claims as follows. The roots of equation (15) are:

$$
\lambda_{1,2}=\frac{1}{2} b^{-1}\left\{1 \pm\left[1+4 b\left(a+c k_{1}\right)\right]^{\frac{1}{2}}\right\}
$$

We can therefore only choose to have real roots (by choosing $k_{1}>-(1+4 a b) / 4 a c$, if $b, c>0$ ); or to have the product of the roots less than one (by setting $k_{1}<(b-a) / c$ in under the same conditions). But we cannot choose the size of the roots individually. In fact to minimize the larger of the two, the best we can do is to set $k_{1}=-a / c$. That will give us $\lambda_{1,2}=\left\{0, b^{-1}\right\}$; which means that we can stabilize the system with a simple feedback rule, but only if the system is already stable $(b>1)$. This case is not of much interest, therefore 
Notice that stabilizability here is determined by the coefficients of the model - not by the policy rule. So it is correct to say that a forward looking New Keynesian model may not be stabilized by a Taylor rule. But if instability follows, the fault lies not with the rule, but with the model. Taylor rules do not, in themselves, destabilize the economy.

By contrast, if we use a forward looking rule like (12), the controlled economy will behave as in equation (16). The roots of this system are:

$$
\lambda_{1,2}=\frac{1}{2}(b+c d)^{-1}\left\{1 \pm\left[1+4(b+c d)\left(a+c k_{1}\right)\right]^{1 / 2}\right\}
$$

which can be set to be arbitrarily close to zero - for example, by selecting i) $d=-b / c+\omega$ where $\omega>1$; and ii) $k_{1}=-a / c$. The system's roots are then $\lambda_{1,2}=\frac{1}{2}\left(\omega^{-1} \pm \omega^{-1}\right)=\left\{0, \omega^{-1}\right\}$, both of which lie within the unit circle. Stability is therefore assured in all possible circumstances. Indeed these roots are arbitrarily small if $\omega$ is made large enough. In this case, therefore, an arbitrary degree of stability can be conferred on any model, including those that were unstable to start with. Moreover, the stabilization is done by the rule, not the model. The key difference, in this case, is that the forward looking component in the rule, $d$, supplies the roots of the stabilized economy.

\section{Conclusions}

In this paper we have outlined a general theory of forward guidance for policymaking. We have used entirely conventional assumptions about an economy under rational expectations, on the model used to represent it, and on the way private sector expectations are formed and can be exploited by the policymaker. We have thus provided a framework to explain the role and strategic advantages of including forward guidance as an explicit policy tool, and to underline the adverse consequences for performance when that guidance is withdrawn. What this paper shows is that forward guidance is an essential component of any policy rule in an economy which is subject to forward looking anticipations of future behavior. 
i) Given the rank condition in theorem 2, forward guidance is a necessary condition to secure stabilizability and/or controllability (the ability to stabilize around and ultimately reach specified values for the target variables).

ii) An economy that is controllable for a certain set of target variables and time horizon, is also stabilizable for those targets and time horizon. And if it is stabilizable, it is controllable. So it is only necessary to test the rank condition in theorem 2 before applying forward guidance.

iii) Without forward guidance that provides the private sector with information about the policy maker's future intentions, the economy may not be stabilizable; and will in general not be controllable with respect to any given target values either.

iv) The stabilizability and controllability properties conferred by forward guidance take effect immediately, from period $t=1$, rather than after $t$ periods delay as would be the case in an economy without anticipations effects.

v) Forward guidance therefore accelerates the required policy impacts. It offers the policymaker the opportunity to control the economy from any date, as the private sector anticipates his future behavior and knows that he can control the economy.

vi) Time inconsistency is not a problem under forward guidance unless there are insufficient policy instruments and policymakers are impatient (have very short horizons). Specifically there is no reason to suppose that policymakers acting in their own interest will show time inconsistent behavior unless both $m<S$ and $S / m<t$, where $t$ is the date by which stability or the desired target values are to be attained.

\section{References}

Acocella, Nicola, and Andrew Hughes Hallett (2014) Forward guidance and controllability. Manuscript, "Sapienza" University of Rome, Rome, Italy.

Acocella, Nicola, Giovanni Di Bartolomeo and Andrew Hughes Hallett (2013) The Theory of Economic Policy in a Strategic Context. Cambridge and New York: Cambridge University Press.

Acocella, Nicola, Giovanni di Bartolomeo, Andrew Hughes Hallett and Paolo Piacquadio (2014) Announcements as an equilibrium selection device. Oxford Economic Papers 66, 325-347. 
Amato, Jeffery D., Stephen Morris and Hyun Song Shin (2002) Communication and Monetary Policy. Oxford Review of Economic Policy 18, 495-503.

Blanchard, Olivier Jean and Charles M. Khan (1980) The Solution of Linear Difference Models under Rational Expectations. Econometrica 48, 1305-1310.

Brand, Claus, Daniel Buncic and Jarkko Turunen (2010) The Impact of ECB Monetary Policy Decisions and Communication on the Yield Curve. Journal of the European Economic Association 8, 1266-1298.

Campbell, Jeffrey R., Charles Evans, Jonas D. M. Fisher and Alejandro Justiniano (2012) Macroeconomic Effects of Federal Reserve Forward Guidance. Brookings Papers on Economic Activity Spring, 1-80.

Cochrane, John H. (2011) Determinancy and Identification with Taylor Rules. Journal of Political Economy 119, 565-615.

Coeuré Benoit (2013) The usefulness of forward guidance. Speech to the Money Marketers Club of New York, European Central Bank, 26 September.

Contessi, Silvio and Li Li (2013) Forward Guidance 101A: A Roadmap of the U.S. Experience. Federal Reserve Bank of St. Louis, Economic Synopses No. 25, September.

Del Negro, Marco, Marc Giannoni and Christina Patterson (2013) The Forward Guidance Puzzle. Staff Report 574, Federal Reserve Bank of New York.

Filardo, Andrew and Boris Hofmann (2014) Forward guidance at the zero lower bound. BIS Quarterly Review, March: 37-53.

Gagnon, Joseph, Matthew Raskin, Julie Remarche and Brian Sack (2011) The Financial Market Effects of the Federal Reserve's Large Scale Asset Purchases. International Journal of Central Banking 7, 3-43.

Gavin, William T., Benjamin D. Keen, Alexander W. Richter and Nathaniel A. Throckmorton (2013) The stimulative effect of forward guidance. Working Paper 2013038A, Federal Reserve Bank of St Louis.

Hughes Hallett, Andrew and Paul G. Fisher (1988) Efficient Solution Techniques for Linear and Nonlinear Rational Expectations Models. Journal of Economic Dynamics and Control 12, 635-657.

Hughes Hallett, Andrew, Nicola Acocella, and Giovanni Di Bartolomeo (2012a) A General Theory of Controllability and Expectations Anchoring for Small Open Economies. Journal of International Money and Finance 31, 397-411.

Hughes Hallett, Andrew, Nicola Acocella, and Giovanni Di Bartolomeo (2012b) Expectations Dynamics: Policy Announcements and the Limits to Dynamic Inconsistency. Studies in Nonlinear Dynamics and Econometrics 16.

Hughes Hallett, Andrew and Nicola Acocella (2014) Rational Expectations, Forward Guidance and Endogenous Policy Rules: Necessary Conditions for Stabilisation, especially at the zero lower bound. Mimeo, University of Rome and St Andrews University.

Hughes Hallett, Andrew, Rasmus Kattai and John M. Lewis (2012) How Reliable Are Cyclically Adjusted Budget Balances in Real Time? Contemporary Economic Policy 30, $75-92$.

Joyce, Michael, David Miles, Andrew Scott and Dimitri Vayanos (2012) Quantitative Easing and Unconventional Monetary Policy. Economic Journal 122, F271-288. 
Kool, Clemens J.M. and Daniel L. Thornton (2012) How Effective Is Central Bank Forward Guidance. Working Papers 2012-063, Federal Reserve Bank of St. Louis.

Levin, Andrew, David Lopez-Salido, Edward Nelson and Tack Yun (2010) Limitations on the Effectiveness of Forward Guidance at the Zero Lower Bound. International Journal of Central Banking 6, 143-89.

Mishkin, Frederic (2002) The Role of Output Stabilization in the Conduct of Monetary Policy. International Finance 5, 213-27.

Raskin, Matthew (2013) The Effects of the Federal Reserve's Data-Based Forward Guidance. Finance and Economics Discussion Series No. 2013-37, Board of Governors, Washington DC, May.

Ugai, Hiroshi (2007) Effects of the Quantitative Easing Policy: A Survey of Empirical Analysis. Monetary and Economic Studies (Bank of Japan) 25, 1-48.

Williams, John C. (2011) Unconventional Monetary Policy: Lessons from the past three years. Federal Reserve Bank of San Francisco, Economic Letter No. 31, October.

Wonham, W. Murray (1974) Linear Multivariable Control. New York, Springer Verlag. 